\title{
Análise reflexiva sobre o desempenho do enfermeiro como mediador da assistência em saúde
}

\author{
Rulio Glécias Marçal da Silva, M.Sc.*, Vagner Ferreira do Nascimento**, Marli Reinado Barbosa, M.Sc.***, \\ Erica Costa dos Santos****, Janielson dos Santos****, Maria Aparecida Pires Almeida****
}

\begin{abstract}
*Enfermeiro, Docente da Faculdade Sequencial, São Paulo/SP, **Enfermeiro, Doutorando em Informação e Comunicação em Saúde pela Fundação Oswaldo Cruz (FIOCRUZ), Docente Assistente da Universidade do Estado de Mato Grosso (UNEMAT), Departamento de Enfermagem de Tangará da Serra, Conselheiro do COREN MT, ${ }^{* *}$ Enfermeira, Coordenadora do Curso de Enfermagem da Faculdade Maú, Mauá/SP e Docente da Faculdade Sequencial, São Paulo/SP, ${ }^{* * * *}$ Graduandos em Enfermagem, Faculdade Sequencial, São Paulo/SP
\end{abstract}

\section{Resumo}

Introdução: Ao considerar que o cuidado é a marca e o núcleo do processo de trabalho de enfermagem, entende-se que as atividades gerenciais do enfermeiro devem ter como finalidade a qualidade do cuidado de enfermagem. Objetivo: Refletir sobre o desempenho do enfermeiro como mediador da assistência em saúde. Método: Trata-se de um estudo de análise reflexiva fundamentada em uma revisão bibliográfica realizada em artigos científicos, livros e capítulos de livros, nas bases de dados Lilacs e BDENF. Resultados: O gerenciamento do cuidado é tratado como a expressão mais clara da boa prática de enfermagem, momento no qual há articulação entre as dimensões gerencial e assistencial para atender às necessidades de cuidado dos pacientes e, ao mesmo tempo, da equipe de enfermagem e da instituição. Cabe ressaltar que o maior risco para o paciente internado é o cuidado fragmentado e o agir alienado dos profissionais. Conclusão: Percebeu-se que o processo de implantação, assim como a escolha de um referencial teórico e uma metodologia adequada, ainda se constitui um grande desafio, principalmente para as lideranças dispostas a desencadear o processo.

Palavras-chave: Enfermagem, gerenciamento em enfermagem, sistematização da assistência de enfermagem.

\section{Abstract}

\section{Reflective analysis of the nurse's role as mediator of health care}

Introduction: Considering that caring is the essence of nursing's work process, it is understood that the management activities of nurses should be directed towards the quality of nursing care. Objective: To reflect on the nurse's role as mediator of health care. Method: This is a reflective analysis study based on a literature review of scientific articles, books and book chapters, in the databases Lilacs and BDENF. Results: The care management is treated as the clearest expression of good nursing practice. There is coordination between management and care dimensions to meet the care needs of patients as well as nursing staff and institution. It is noteworthy that the highest risk for hospitalized patient is the fragmented care and the alienated acting of professionals. Conclusion: It was observed that the implementation process as well as 
the choice of a theoretical framework and appropriate methodology still poses a major challenge, especially for leaders willing to start the process.

Key-words: nursing, nursing management, systematization of nursing care.

\section{Resumen}

\section{Análisis reflexivo sobre el desempeño del profesional de enfermería como mediador de la asistencia sanitaria}

Introducción: Teniendo en cuenta que la atención es la marca y el núcleo del proceso de trabajo de enfermería, se entiende que las actividades de gestión del enfermero (a) deben ser dirigidas hacia la calidad de la atención de enfermería. Objetivo: Reflexionar sobre el papel del profesional de enfermería como mediador de la atención sanitaria. Método: Se trata de un estudio de análisis reflexivo sobre la base de una revisión bibliográfica de artículos científicos, libros y capítulos de libros, en las bases de datos Lilacs y BDENF. Resultados: La gestión de la atención se trata como la expresión más clara de la buena práctica de la enfermería, en la que existe una coordinación entre las dimensiones de gestión y de atención para satisfacer las necesidades de atención de los pacientes y, al mismo tiempo, al personal de enfermería e institución. Cabe resaltar que el mayor riesgo para el paciente hospitalizado es la atención fragmentada y alienación en la práctica profesional. Conclusión: Se observó que el proceso de implementación, así como la elección de un marco teórico y una metodología adecuada, representa todavía un gran desafío, especialmente para los líderes dispuestos a iniciar el proceso.

Palabras-clave: Enfermería, gestión de enfermería, sistematización de la atención de enfermería.

\section{Introdução}

A posição de gerente da assistência de enfermagem e da organização institucional atribuída ao profissional enfermeiro vem sendo investigada, no Brasil, desde os anos 1980 e pesquisa recente confirma a ênfase no trabalho gerencial do enfermeiro, em especial, com base na concepção de gerenciamento do cuidado [1].

Considerando a experiência de enfermeiros na prática profissional, a mesma têm se mostrado com bom desempenho na assistência, mas uma fragilidade na administração de enfermagem ou o inverso, o que expressa que há uma dificuldade de articulação entre as dimensôes gerencial e assistencial. Percebe-se que o enfermeiro que está na administração tende a valorizar esta ação como uma ação que subsidia a viabilização do cuidado; por outro lado, quem está no cuidado tende a menosprezar a atividade do gerenciamento, atribuindo-lhe um cunho burocrático [1].

Ao considerar que o cuidado é a marca e o núcleo do processo de trabalho de enfermagem, entende-se que as atividades gerenciais do enfermeiro deveriam ter como finalidade a qualidade do cuidado de enfermagem, de modo que a cisão entre a dimensão assistencial e gerencial compromete essa qualidade e gera conflitos no trabalho do enfermeiro, seja do profissional com a sua própria prática, seja na sua relação com a equipe de enfermagem e a equipe de saúde [2].

A constituição histórica da enfermagem no interior do sistema hospitalar divide-se em dois extremos. De um lado a busca por assegurar o bom funcionamento da instituição e da ordem médica e, de outro, a busca pela prestação de cuidados contínuos aos pacientes nas 24 horas, o que permitiu a esses profissionais configurar um saber fazer assistencial e de coordenação da assistência. Este saber, à medida que se consolida como possibilidade de articulação, permite um distanciamento da concepção burocrática do gerenciamento e aproximação de uma prática gerencial articuladora e integradora dos processos de trabalho de enfermagem. Dessa forma, "o distanciamento entre o administrar e o cuidar vivenciado no cotidiano de trabalho vem gerando inquietaçôes pessoais e profissionais, e impondo um repensar da prática administrativa voltada à assistência, procurando resgatar o papel do enfermeiro como gerente do cuidado" [3].

A primeira condição para que uma pessoa possa assumir um ato comprometido está em ser capaz de agir e de refletir o que se traduz em um compromisso para a mudança, ou seja, para que o enfermeiro possa assumir uma prática comprometida a partir da Sistematização da Assistência de Enfermagem (SAE), deve ser capaz de refletir e de reconhecer as barreiras históricas e culturais do ensino formal, 
baseadas, mais especificamente, no modelo técnico-burocrático e, dessa maneira, desencadear um processo definitivo de mudança no seu papel [4].

Nesse sentido, a SAE se transforma em um processo dinâmico, capaz de avaliar e de indicar intervençóes contínuas nas açóes da equipe de enfermagem e estreitar os laços profissionais entre a equipe multiprofissional [5].

As dificuldades apontadas pelos enfermeiros relacionadas à implementação, à operacionalização e ao acompanhamento periódico e direto das atividades, bem como a falta de pessoal, o desconhecimento da Lei do Exercício Profissional, a falta de liderança, a falta de comprometimento e a falta de tempo, são fatores que, facilmente, podem resultar em perda de estímulo por parte dos enfermeiros e, consequentemente, gerar desmotivação e insatisfação quanto à realização da SAE [5].

Desse modo, a SAE está diretamente relacionada a uma questão de prioridade e/ou de valorização daquilo que julgamos importante e essencial para a profissão. No entanto, é comum que as maiores dificuldades relacionadas à implementação do processo estejam associadas à descrença e à rejeição dos próprios enfermeiros que se limitam ao modelo técnico-burocrático para não participarem do processo. É preciso compreender, entretanto, que a própria rejeição e inflexibilidade podem caracterizar a falta de um conhecimento específico e a desatualização profissional [5].

A organização da assistência de enfermagem, prestada ao indivíduo, família ou comunidade, mediante utilização das fases do método científico, trata-se de uma terminologia que pode não se adequar ao fenômeno, uma vez que sistematizar pode significar organizar, mas náo necessariamente usando todas as etapas do método científico [6].

A Sistematização da Assistência de Enfermagem (SAE) é uma metodologia de organização, planejamento e execução de açóes sistematizadas que são realizadas pela equipe durante o período em que o indivíduo se encontra sob a assistência de enfermagem. Essa metodologia foi introduzida, inicialmente nas décadas de 1920 e 30, nos cursos de enfermagem, particularmente no ensino dos estudos de caso e no planejamento de cuidados individualizados. No Brasil, a SAE começou a ser implantada nos serviços de enfermagem nas décadas de 1970 e 80, fortemente influenciada por Wanda de Aguiar Horta [7].
A SAE, enquanto processo organizacional é capaz de oferecer subsídios para o desenvolvimento de métodos/metodologias interdisciplinares e humanizadas de cuidado. Ou seja, é a organização do trabalho, segundo as fases do seu fluxo. Implica na definição da natureza e do tipo do trabalho a ser realizado, desde a base teórico-filosófica, o tipo de profissional requerido, técnicas, procedimentos, métodos, objetivos e recursos materiais para a produção do cuidado. Sua aplicação nas instituições de saúde apresenta muitos aspectos positivos entre eles: segurança no planejamento, execução e avaliação das condutas de enfermagem, a individualização da assistência, visibilidade e autonomia para o enfermeiro, diminuição do tempo de hospitalização e consequentemente economia de recursos [7].

O objetivo desse trabalho é fazer uma reflexão sobre o desempenho do enfermeiro como mediador da assistência em saúde.

\section{Métodologia}

Estudo descritivo, de análise reflexiva fundamentada em uma revisáo bibliográfica realizada em artigos científicos, livros e capítulos de livros, nas bases de dados Lilacs (Literatura Latino-Americana e do Caribe em Ciências da Saúde) e BDENF (Base de Dado de Enfermagem), no endereço eletrônico: www.bireme.br e manualmente na Biblioteca da Faculdade Sequencial. Após a leitura prévia dos resumos dos trabalhos levantados, foram selecionados os pertinentes ao objetivo deste estudo.

Os trabalhos eleitos foram fichados e agrupados quanto ao assunto, título, ano de publicação, editora, autores, citaçóes relevantes à pesquisa, havendo ao final a produçáo da revisão reflexiva.

Foram critérios para inclusão os artigos que incluíssem os termos e descritores: enfermagem, dimensionamento de pessoal de enfermagem, recursos humanos, gerenciamento em enfermagem, entre os anos de 2005 a 2013, de língua portuguesa e de acesso livre.

\section{Resultados e discussão}

A avaliação de desempenho do enfermeiro se faz por meio de requisitos administrativos e do cuidado. Esse último se faz por meio do Processo de Enfermagem figurado pela Sistematização da Assistência de Enfermagem. Em tempos atuais, em que os padróes de qualidade são inerentes aos serviços 
de enfermagem, uma boa assistência de enfermagem é indiscutível.

Os estudos revelam que existe o sentimento de insegurança dos profissionais para realizar as atividades inerentes ao Processo de Enfermagem, por náo dominá-las adequadamente, o que conduz à desvalorização desse método de organização do cuidar pela própria categoria. Ainda, na maioria dos serviços de Enfermagem, restrição a um modelo que se volta predominantemente para a execução das açóes prescritas pelo profissional médico, alia$\mathrm{da}$ ao fato de que na maioria das vezes o número de enfermeiros é insuficiente para a implantação e desenvolvimento de uma sistematização efetiva e de qualidade $[8,9]$.

Obstáculos para a implementação e desenvolvimento do Processo de Enfermagem se figuram em todos os setores de atendimento aos usuários e este quadro muita das vezes se dá pela falta de tempo disponível para a realização deste método de assistência [10].

Aliado a isso o gerenciamento em enfermagem, especialmente no ambiente hospitalar, sofre forte influência da administração clássica, em particular do modelo taylorista/fordista e burocrático, caracterizado por divisão do trabalho, hierarquia, autoridade legal, sistema de procedimentos e rotinas, impessoalidade nas relaçóes interpessoais e outros [11].

O gerenciamento do cuidado é, dessa forma, tratado como a expressão mais clara da boa prática de enfermagem, momento no qual há articulação entre as dimensóes gerencial e assistencial para atender às necessidades de cuidado dos pacientes e ao mesmo tempo da equipe de enfermagem e da instituição. Cabe ressaltar, nessa particularidade, que o maior risco para o paciente internado é o cuidado fragmentado e o agir alienado dos profissionais. Considera-se alienação o estranhamento do profissional em relaçáo ao produto ou resultado do seu próprio trabalho, no qual ele náo reconhece a sua contribuição [12].

Apesar das consideraçóes citadas sobre a importância dos aspectos legais e assistenciais, pesquisas revelam que com muita frequência as anotaçóes da enfermagem não contêm as informaçóes necessárias para apoiar a instituição e/ou a enfermagem, no caso de um processo judicial. Os registros no prontuário do paciente são de enorme importância, mas há quase descaso quanto a esse tipo de formalização escrita de trabalho, e a falta de anotação no prontuário do paciente muitas vezes dificulta o exercício da proteção dos direitos dos profissionais de enfermagem, quer judicialmente, quer administrativamente $[13,14]$.

Foi identificado por um estudo intra-hospitalar pautado em uma concepçáo ampliada de saúde e doença que contempla náo apenas a produção baseada no modelo biomédico, mas a dimensão ontológica e existencial dos sujeitos, portanto abarcando a intersubjetividade, que a prevalência de conhecimentos e práticas biomédicas tem-se sobreposto não somente a proposta metodológica do processo de enfermagem, mas também ao conceito de cuidado ampliado adotado pelos autores que o entendem como "uma relaçáo que se estabelece para os sujeitos e entre os sujeitos, de acordo com as suas necessidades e não somente com as do profissional, $\mathrm{o}$ que caracteriza o cuidado como o núcleo do trabalho de enfermagem" [15].

Para que a SAE represente a conquista de um "novo espaço", isto é, um novo modo de pensar, fazer, ensinar e gerenciar as mudanças, a prática de enfermagem necessita ser questionada à luz de metodologias problematizadoras, para que a sua trajetória, enquanto um processo cultural, náo se constitua em mais uma alternativa frustrada e/ou um processo puramente normativo e/ou legal [16].

Nesse sentido, a SAE representa a construção dinâmica de um novo espaço cultural voltado para um sistema de valores que pressupóe a superaçáo constante da descrença e do conformismo, da fragmentação e da burocratização, a fim de estimular a liberação da criatividade e da subjetividade, tendo em vista uma nova percepçáo da realidade, ou seja, uma mudança no papel do enfermeiro passível de ser avaliada até mesmo do ponto de vista de desempenho do profissional [16].

É unânime a percepção de que as tentativas de implantar a SAE nem sempre atingem o êxito desejado, pois "esbarram" em dificuldades das mais variadas origens, tornando a implantaçáo da SAE um processo desestimulador e muitas vezes inviável na prática dos profissionais de enfermagem corroborando um mau desempenho do profissional nesse aspecto [17].

O enfermeiro, para prestar a assistência de enfermagem com qualidade e humanismo, necessita inserir-se na realidade concreta de forma consciente, competente, técnica e científica. Dessa forma, a implantação da Sistematização da Assistência de Enfermagem (SAE), a partir de um conhecimento 
específico e de uma reflexão crítica acerca da organização e da filosofia do trabalho de enfermagem, constitui-se um instrumento de fundamental importância para que o enfermeiro possa gerenciar e otimizar a assistência de enfermagem de forma organizada, segura, dinâmica e competente [18].

No entanto o que se percebe é que mesmo após anos de sua criaçáo e oferecendo tantas vantagens para o profissional e o indivíduo, a implantação da SAE ainda náo ocorreu a contento e isso se dá devido a uma série de obstáculos que necessitam ser vencidos como: a falta de reconhecimento por parte da equipe de enfermagem, o número de enfermeiros nos serviços, o envolvimento com o processo, a valorização por parte da administraçáo da instituição, bem como os indicadores de resultado da assistência. Além disso, uma sólida base científica, conhecimento, habilidades e atitudes pautadas no compromisso ético, na responsabilidade e no assumir o cuidado do outro [18].

\section{Conclusão}

Percebeu-se que o processo de implantação, assim como a escolha de um referencial teórico e uma metodologia adequada, ainda se constitui um grande desafio, principalmente para as lideranças dispostas a desencadear o processo.

Carecemos de uma maior valorização, a começar na formação do profissional dessa que é uma atividade única e exclusivamente privativa do enfermeiro e que pode melhorar acentuadamente sua avaliação de desempenho em sua verdadeira essência, o cuidado.

\section{Referências}

1. Peduzzi M, Hausmann M. Articulação entre as dimensões gerencial e assistencial do processo de trabalho do enfermeiro. Texto Contexto Enferm 200918(2):25865.

2. Silva KL, Sena RR. A formação do enfermeiro: construindo a integralidade do cuidado. Rev Bras Enferm 2006;59(4):488-91.

3. Trevizan MA, Mendes IAC, Melo MRAC. A busca da competência do cuidado segundo Boff: uma nova perspectiva de conduta ética da enfermeira gerente. Rev Latinoam Enferm 2003;11(5):652-7.

4. Lunardi VL, Lunardi Filho WD, Silveira RS, Soares NV, Lipinski JM. O cuidado de si como condição para o cuidado dos outros na prática de saúde. Rev Latinoam Enferm 2004;12(6):933-9.

5. Backes DS, Schwartz E. Implementação da sistematização da assistência de Enfermagem: desafios e conquistas do ponto de vista gerencial. Ciência Cuidado e Saúde 2005;4(2):182-188.

6. Takahashi AA. Dificuldades e facilidades apontadas por enfermeiras de um hospital de ensino na execução do processo de enfermagem. Acta Paul Enferm 2008;21(1):32-8.

7. Marques BL, Houstoun CJ. Administração e liderança em enfermagem: teoria e aplicação. 2 ed. Porto Alegre: Artes Médicas; 1999.

8. Brandalize DL, Kalinowski CE. Processo de enfermagem: vivência na implantação da fase de diagnóstico. Cogitare Enferm 2005;10(3):53-7.

9. Pivotto F, Filho WDA, Lunardi VL. Prescrição de enfermagem: dos motivos da náo realização as possíveis estratégias de implementação. Cogitare Enferm 2004;9(2):32-42.

10. Backes DS, Esperança MP, Amaro AM, Campos IEF, Cunha ADC, Schwartz E. Sistematizaçáo da assistência de enfermagem: percepçáo dos enfermeiros de um hospital filantrópico. Revista Acta Sci Health Sci 2005;27(1):25-9.

11. Cogo E, Gehlen MH, Ilha S, Zamberlan C, Freitas HMB, Backes DS. Sistematização da assistência de enfermagem no cenário hospitalar: percepção dos enfermeiros. Cogitare Enferm 2012;17(3):513-8.

12. Gonçalves LRR. O desafio de implantar a sistematizaçáo da assistência de enfermagem sob a ótica de discentes. Escola Anna Nery Rev Enferm 2007;11(3):459-65.

13. Hermida PMV. Desvelando a implementação da sistematização da assistência de enfermagem. Rev Bras Enferm 2004;57(6):733-7.

14. Padoin SMM, Mello SMM, Neves ET, Ribeiro AC, Tronco CS. A sistematização da assistência de enfermagem na perspectiva da equipe de um hospital de ensino. Rev Eletr Enferm 2010;12(4):655-9.

15. Remizoski J, Rocha MM, Vall J. Dificuldades na implantação da sistematização da assistência de enfermagem - SAE: uma revisão teórica. Cad Saúde Pública 2010;3:1-14.

16. Lopes FL, Szewczyk MSC, Lunardi VL, Santos SSC. SAE como um novo fazer na atividade cuidativa da enfermeira com base na complexidade de Edgar Morin. Rev Cogitare Enferm 2007;12(1):109-13.

17. Reppetto MA, Souza MF. Avaliaçáo da realizaçáo e do registro da sistematizaçáo da assistência de enfermagem (SAE) em um hospital universitário. Rev Bras Enferm 2005;58(3):325-9.

18. Castilho NC, Ribeiro PC, Chirelli MQ. A implementação da sistematizaçáo da assistência de enfermagem no serviço de saúde hospitalar do Brasil. Texto Contexto Enferm 2009;18(2):280-9.

19. Thomas VA, Guidardello EB. Sistema da Assistência de Enfermagem. Problemas identificados pelos enfermeiros. Nursing 2002;54:28-34. 